\title{
Sustained Release of Multiple Growth Factors from Injectable Polymeric System as a Novel Therapeutic Approach Towards Angiogenesis
}

\author{
Qinghua Sun, ${ }^{1,2,3}$ Eduardo A Silva, ${ }^{4}$ Aixia Wang, ${ }^{3}$ James C. Fritton, ${ }^{5}$ David J. Mooney, ${ }^{4}$ Mitchell B. Schaffler, ${ }^{5}$ \\ Paul M. Grossman, ${ }^{6}$ and Sanjay Rajagopalan, ${ }^{2,3,7}$
}

Received August 27, 2009; accepted November 16, 2009; published online December 1, 2009

\begin{abstract}
Purpose. The aim was to investigate that a bio-degradable alginate and poly lactide-co-glycolide (PLG) system capable of delivering growth factors sequentially would be superior to single growth factor delivery in promoting neovascularization and improving perfusion.

Methods. Three groups of apoE null mice underwent unilateral hindlimb ischemia surgery and received ischemic limb intramuscular injections of alginate (Blank), alginate containing $\mathrm{VEGF}_{165}$ (VEGF), or alginate containing VEGF $_{165}$ combined with PLG microspheres containing PDGF-BB (VEGF/PDGF). Vascularity in the ischemic hindlimb was assessed by morphologic and immunohistochemical end-points, while changes in blood flow were assessed by Laser Doppler Perfusion Index. Muscle VEGF and PDGF content was assessed at multiple time points.

Results. In the VEGF/PDGF group, local tissue VEGF and PDGF levels peaked at week 2 and 4 , respectively, with detectable PDGF levels at week 6 . At week 6 , mean vessel mean diameter was significantly greater in the VEGF/PDGF group compared to the VEGF or Blank groups with evidence of well-formed smooth muscle-lined arterioles.

Conclusions. Sequential delivery of VEGF and PDGF using an injectable, biodegradable platform resulted in stable and sustained improvements in perfusion. This sustained, control-released, injectable alginate polymer system is a promising approach for multiple growth factor delivery in clinical application.
\end{abstract}

KEY WORDS: alginate; angiogenesis; PDGF; polymer; VEGF.

\section{INTRODUCTION}

Neovascularization in adults results not only from angiogenesis but also from vasculogenesis in which the sprouting and growth of small vessels along with the branching and extension of existing capillaries by the assembly of endothelial cells from preexisting vessels develop. Until recently,

\footnotetext{
${ }^{1}$ Division of Environmental Health Sciences, College of Public Health, The Ohio State University, Columbus, Ohio 43210-1252, USA.

${ }^{2}$ Division of Cardiovascular Medicine, College of Medicine, The Ohio State University, Columbus, Ohio 43210-1252, USA.

${ }^{3}$ Davis Heart \& Lung Research Institute, College of Medicine, The Ohio State University, 460W 12th Avenue, Room 398, Columbus, Ohio 43210-1252, USA.

${ }^{4}$ School of Engineering and Applied Sciences, Harvard University, Cambridge, Massachusetts, USA.

${ }^{5}$ New York Center for Biomedical Engineering, The City University of New York, New York, New York, USA.

${ }^{6}$ Division of Cardiovascular Diseases, Department of Internal Medicine, University of Michigan, Ann Arbor, Michigan 481090306, USA.

${ }^{7}$ To whom correspondence should be addressed. (e-mail: sanjay. rajagopalan@osumc.edu)
}

vascular endothelial growth factor (VEGF) was proven to be specific and critical for blood vessel formation (1). Recent reports suggest that local administration of VEGF significantly increases myocardial perfusion by adenovirus and improvement in angina and favorable trends in exercise treadmill test time and angina frequency by recombinant protein (2,3). Platelet-derived growth factor (PDGF), which recruits smooth muscle cells and pericytes to promote extracellular matrix deposition to stabilize neovessels, has recently been demonstrated to result in larger, mature vessel formation, especially if delivered following VEGF $(4,5)$.

Although growth factors clearly play important roles in angiogenesis and arteriogenesis, the appropriate mode for making these factors available at the desired site with desired dosage for a desired period of time remains unclear. Therapeutic angiogenesis via adenovirus has significant limitations, such as biologically brief activity or expression (less than 3 weeks). Bolus delivery of protein molecules leads to undesirable systemic effects and toxicity and an insufficient local concentration for the required time-frame due to the rapid degradation and subsequent distribution throughout the body. Therefore, a new platform to deliver growth factor in an optimized, controlled pattern is absolutely needed. We have developed a polymeric growth factor delivery vehicle in which dual delivery of VEGF and PDGF resulted in a rapid formation of a mature vascular network. This structural 
polymer scaffold system is capable of delivery of multiple angiogenic factors with distinct kinetics and is potentially applicable for the patients from diabetic or other ischemic peripheral diseases.

In this study, we tested the hypothesis that dual delivery of VEGF and PDGF, in a controlled, sequential pattern of injectable gel form, can direct the formation of a mature vasculature, as compared to the delivery of VEGF alone. The system developed to deliver multiple growth factors may find broad utility in the clinical application of peripheral and cardiovascular ischemic diseases.

\section{MATERIALS AND METHODS}

\section{Alginate Fabrication and Analysis of Release Kinetics In Vitro}

The details of the fabrication were reported previously (6). Briefly, alginate hydrogels were formed from a $2 \%(w / w)$ solution of alginate polymer. The alginate (ProNova MVG; ProNova Polymers, Norway) used to form gels was a combination of two different molecular weight polymers $(250,000$ and 60,000 Dalton) at a ratio of $6: 4$. Both alginate polymers were oxidized to a degree of $1 \%$ prior to gel formation to create sites in the polymer that were susceptible to degradation in aqueous medium. To prepare gels, the $2 \%$ alginate solutions were combined with a calcium solution (40 $\mu \mathrm{l}$ of $\mathrm{Ca}^{2+}$ per $1 \mathrm{ml}$ of $2 \%$ alginate solution) using a $1 \mathrm{ml}$ syringe. In the experiment using PDGF delivery, the growth factor was first pre-encapsulated in PLG microspheres that were combined with the alginate solution prior to gelling. These microspheres were prepared using a double emulsion process as previously described (7). Alginates were fabricated with ${ }^{125}$ I-VEGF or ${ }^{125}$ I-PDGF as tracers for in vitro release studies, and these studies were performed as previously described (4).

\section{Animals and Surgical Procedures}

Male $\mathrm{apoE}^{-/-}$mice (Jackson Laboratories, Bar Harbor, $\mathrm{ME})$ aged 6 weeks were used in this study. $\mathrm{ApoE}^{-/-}$mice were fed a high fat diet ( $21 \%$ fat, $0.15 \%$ cholesterol, Harlan Teklad) for at least 6 weeks prior to enrollment in the study. Animals were handled according to protocols approved by the University of Michigan Committee on Use and Care of Animals and cared for according to standards in the Guide for the Care and Use of Laboratory Animals. Mice were anesthetized with an intraperitoneal injection of a mixture of ketamine $80 \mathrm{mg} / \mathrm{kg}$ and xylazine $5 \mathrm{mg} / \mathrm{kg}$ prior to all surgical procedures. Unilateral hindlimb ischemia was induced as previously described $(5,8)$. After the vessel ligation, 45 mice were randomized into one of the three groups: alginate solution alone, alginate with VEGF, or alginate with VEGF plus PDGF. Four separate injections of total volume $50 \mu \mathrm{l}$ of alginate solutions, or alginate solutions with microspheres containing $3 \mu \mathrm{g} \mathrm{VEGF}_{165}$, or alginate solutions with microspheres containing $3 \mu \mathrm{g} \mathrm{VEGF}_{165}$ plus $3 \mu \mathrm{g}$ PDGF $_{\mathrm{BB}}$, were performed directly into the area where the vessels were ligated with a $25 \mathrm{G}$ needle (Becton Dickinson) immediately after the vessel ligation. The incision was surgically closed, and the mice were observed up to 6 weeks.

\section{Laser Doppler Perfusion Imaging}

Hindlimb perfusion was measured using a laser Doppler perfusion imaging (LDPI) system (Lisca Inc, Sweden). Consecutive measurements were obtained by scanning of the region of interest (legs and feet) on anesthetized animals. The stored perfusion values were retrieved for analysis. To account for variables such as ambient light and temperature, the results are expressed as the ratio of perfusion in the left (ischemic) versus right (normal) limb (5).

\section{Microcomputed Tomography Imaging}

After anesthesia and thoracotomy, left ventricle was catheterized and injected with heparinized saline $(0.9 \%$ sodium chloride with 5,000 U of heparin) at an appropriate pressure in order to clear the cardiovascular network of the remaining blood by right atrium opening. A low-viscosity, radiopaque liquid polymer compound (MV-122, Flow Tech Inc., Carver, MA) was then injected through the cannulae, and the hindlimbs were then immersed in $10 \%$ buffered formalin and placed under refrigeration at $4{ }^{\circ} \mathrm{C}$ overnight to allow polymerization of compound, followed by $95 \%$ alcohol solution for one day. At successive 24-h intervals, the glycerin concentration was raised from $30 \%$ to $50 \%$ to $75 \%$, and finally to pure glycerin. Then, the specimen was kept in $10 \%$ formalin for microcomputed tomography (Micro-CT) imaging as described (9). All specimens were traced over their entire length, and cross-sections were analyzed at 1-mm intervals.

\section{Histological Analysis, Immunoassay and Immunohistochemistry}

Mice were sacrificed at predetermined time points (day 1442) after surgery with an overdose of sodium pentobarbital. For total protein and DNA extraction, isolated tissue samples were rinsed in PBS to remove excess blood, snap-frozen in liquid nitrogen, and stored at $-80^{\circ} \mathrm{C}$ until use (10). Recombinant protein concentration in tissue lysate was determined using Quantikine Immunoassay systems for human VEGF and human PDGF BB (R\&D Systems Inc., Minneapolis, MN) according to the manufacturer's instructions. Optical density was measured at $405 \mathrm{~nm}$ with a wavelength correction of $540 \mathrm{~nm}$ using an enzyme-linked immunosorbent assay (ELISA) reader (Spectra Max 340, Molecular Devices Corp., Sunnyvale, CA).

For immunohistochemistry, retrieved scaffolds and muscle tissue from ischemic and nonischemic hindlimbs were fixed in $10 \%$ zinc formalin overnight. Tissues were paraffin embedded and sectioned into $5 \mu \mathrm{m}$-thick sections according to standard procedures. Tissue sections were stained with antibodies for CD31 (BD Biosciences PharMingen, San Diego CA) or $\alpha$-smooth muscle actin ( $\alpha$-SMA, DakoCytomation, Carpinteria, CA). For measurement of capillary density, two different sections were taken $5 \mathrm{~mm}$ apart, and the capillaries counted per 30 randomly chosen high-power fields on the two sections (8). The number of positively stained blood vessels was manually counted and normalized to the tissue area using a light microscope Olympus BH-2 (Tokyo, Japan). To obtain the average vessel cross-sectional area, a minimum of 10 individual images were sampled, and 
at least 100 blood vessels were measured. Images were analyzed using Image Pro-plus 4.0 (Media Cybernetics, CA).

\section{Statistical Analysis}

All values are expressed as mean $\pm \mathrm{SD}$, and were analyzed by one-way ANOVA with a Friedman test using GraphPad Prism software (version 3.00, GraphPad Software Inc., CA). A $p$ value $<0.05$ was considered significant.

\section{RESULTS}

\section{Injectable Polymeric Release System}

Fig. 1 shows the in vitro release kinetics of preencapsulated PDGF and VEGF of alginate fabricated from PLG. Approximately $50 \%$ of the VEGF was released from the alginate within 2 days, and reached more than $70 \%$ by day 12 and more than $80 \%$ by day 36 . In contrast to VEGF, PDGF was released relatively slower, with about $10 \%$ within 2 days, and reached more than $40 \%$ by day 12 and more than $70 \%$ by day 36 (Fig. 2).

\section{Accelerated Recovery of Hindlimb Blood Flow by VEGF/PDGF}

Blood flow was reduced to less than $20 \%$ of the baseline following ligation surgery. There was no significant difference in limb perfusion between groups in the first 2 weeks after the operation. The blood flow remained severely impaired and showed delayed recovery after vessel ligation in the group that received blank alginate solution containing no growth factor. The VEGF/PDGF group of $3 \mu \mathrm{g} \mathrm{VEGF}_{165}$ with $3 \mu \mathrm{g}$ $\mathrm{PDGF}_{\mathrm{BB}}$ recovered significantly faster than the blank or the VEGF group of $3 \mu \mathrm{g} \mathrm{VEGF}_{165}$ and achieved more than $80 \%$ of the initial value by the end of the experiment. VEGF

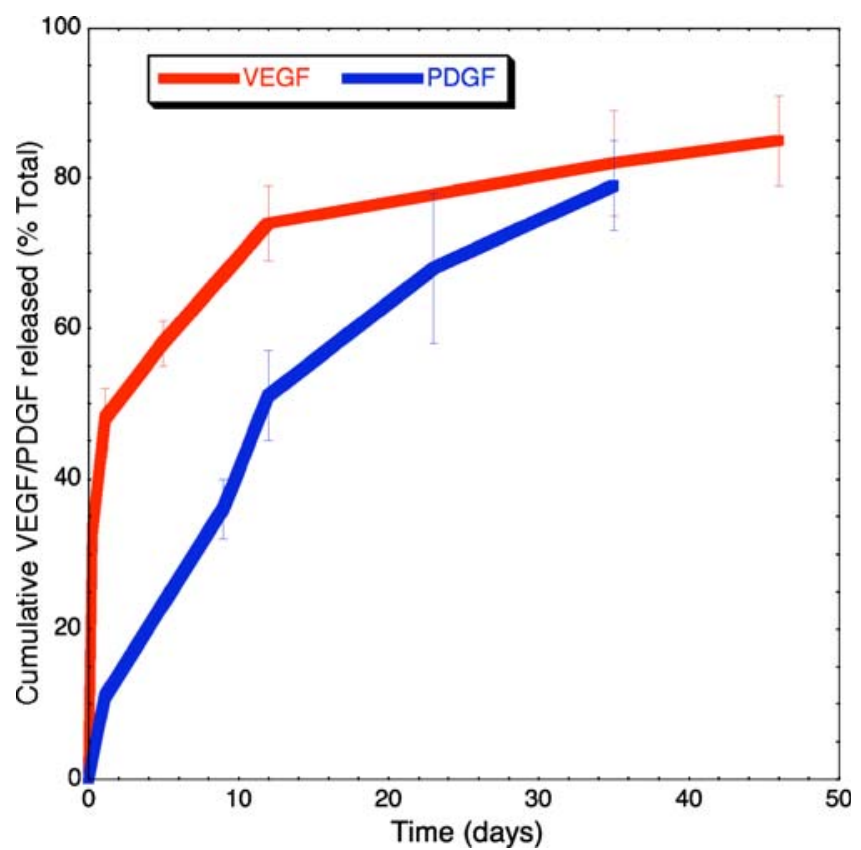

Fig. 1. In vitro release kinetics of pre-encapsulated PDGF and VEGF from alginate fabricated from PLG.

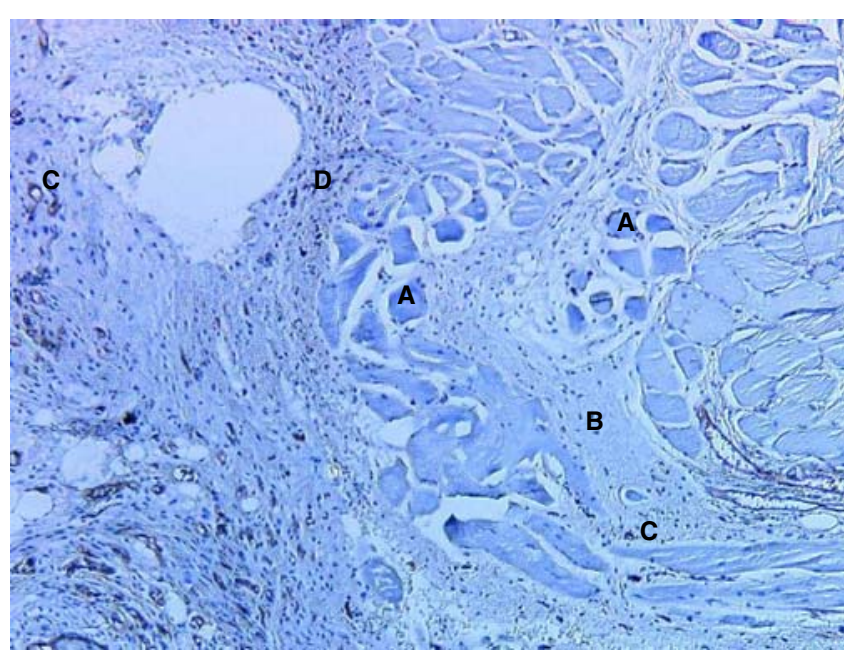

Fig. 2. Representative image of CD 31 staining of thigh muscle treated by intramuscular injection of alginate containing VEGF at week $3, \times 200$. $A$, alginate; $B$, new tissue; $C$, new vessel; $D$, inflammatory cells.

showed a positive effect on the recovery of perfusion from week 3 to week 6 compared to the blank group, but weaker than the VEGF/PDGF group (Fig. 3). Micro-CT scanning showed clear restoration of blood flow in the previously ligated vessels and the filling of collateral vessels by the VEGF or VEGF/PDGF treatment, compared to the blank (Fig. 4).

\section{Increased Capillary Vessel Density by Both VEGF and VEGF/ PDGF Delivery}

A small number of capillary vessels in the muscle were positively stained following the injection of blank alginate solution, even after 6 weeks intervention (Fig. 5, top row). VEGF alone or VEGF/PDGF delivery did not initiate a significant increase in capillary vessel density in the first 2 weeks, but both did induce a statistically significant increase in capillary vessel density from week 3 to week 6 (Fig. 5, middle and bottom rows). In addition, VEGF alone led to the highest density of vessels among the three groups (Fig. 7).

\section{VEGF/PDGF Results in the Formation of Matured Vessels}

To assess the maturity of blood vessels, $\alpha$-SMA was stained on the muscle tissue. We found few positive staining vessels in the blank scaffold group (top row of Fig. 6). With VEGF alginate injection, the number of positive staining vessels increased (middle row of Fig. 6). Sections from the dual delivery of VEGF/PDGF were observed to have much stronger a-SMA staining and larger, thicker vessel formation (bottom row of Fig. 6). The measurement of cross-sectional areas of blood vessels from $\alpha$-SMA-stained thigh muscle of the mice showed a significant increase in mean vessel diameters and a distribution towards larger, mature vessels in VEGF/PDGF group (Fig. 8). In contrast, VEGF resulted in a small increase in vessel diameter (Figs. 7 and 8). 


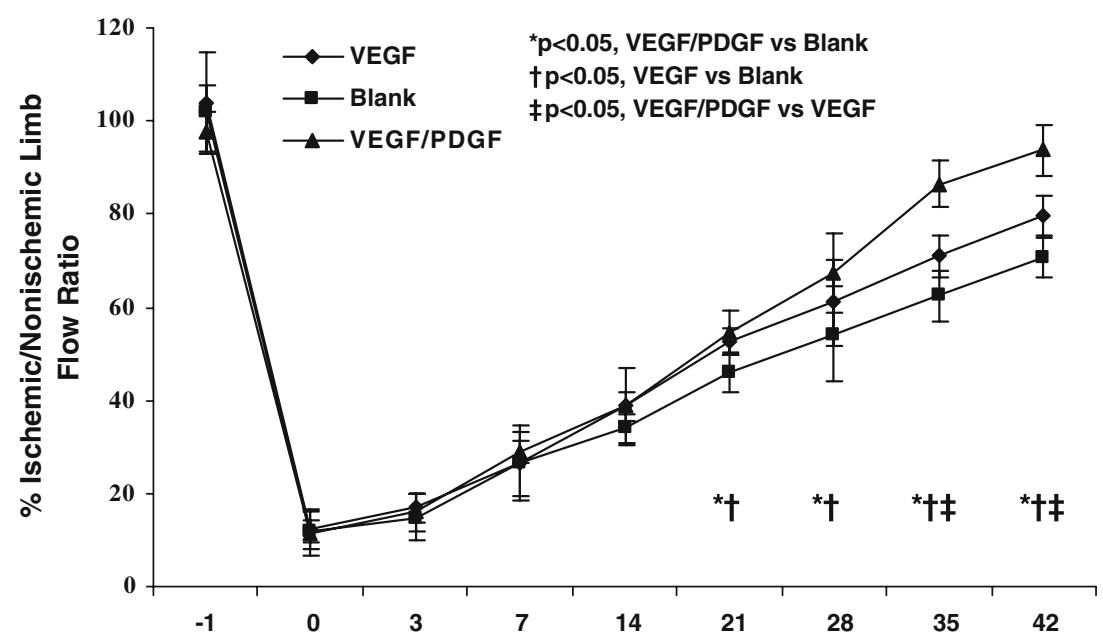

Fig. 3. Computer-assisted quantitative analyses of hindlimb blood flow in apoE ${ }^{-/-}$mice demonstrated significantly enhanced ratio of ischemic to untreated limb blood perfusion in mice ischemic limb receiving intramuscular injections of alginate (Blank), alginate containing $\mathrm{VEGF}_{165}$ (VEGF), or alginate containing $\mathrm{VEGF}_{165}$ combined with PLG microspheres containing PDGF-BB (VEGF/PDGF).

\section{Sustained Growth Factor Delivery}

Verification of sustained growth factor delivery in vivo was accomplished by testing the concentration of VEGF or PDGF from muscle tissue where the injections occurred by ELISA. The concentrations of VEGF or PDGF in the thigh muscle obtained from the VEGF or VEGF/PDGF groups reached a plateau from week 2 to week 4. Unlike human VEGF, human PDGF was still detectable at the end of the experiment at week 6 (Fig. 9).

\section{DISCUSSION}

Decades of experimental studies in a variety of animals have raised the concept that new vessels could be created by growth factor(s) to reduce ischemia despite most of the clinical trials so far being disappointing (11). New blood vessel formation is critical to many cardiovascular diseases. It is reported that $90 \%$ of major amputations are performed for ischemia, $25 \%$ of patients with critical limb ischemia require amputation, and the incidence of critical limb ischemia can be approximately 500 to 1,000 new cases per million persons per year (12). Coronary artery disease is a major health problem for over 12 million Americans, and an increasing number of patients are not candidates for revascularization through traditional surgical methods. The alternative methods that could form new blood vessels via branching from existing vessels bring hope to these patients. In fact, the primary physiological response to tissue ischemia is the local growth of capillaries, and a vast increase in the capillary bed size is

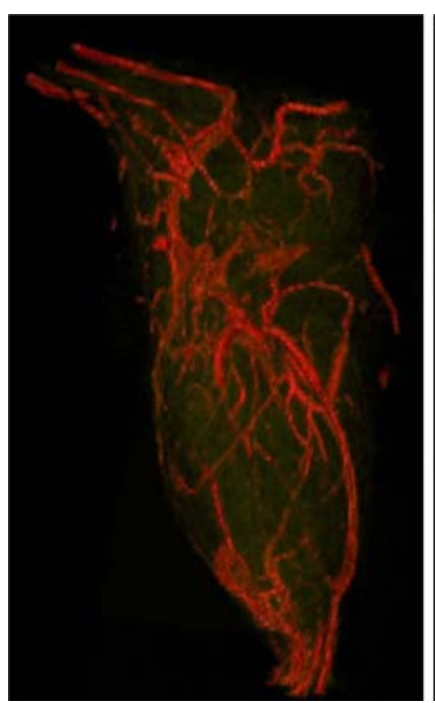

Blank

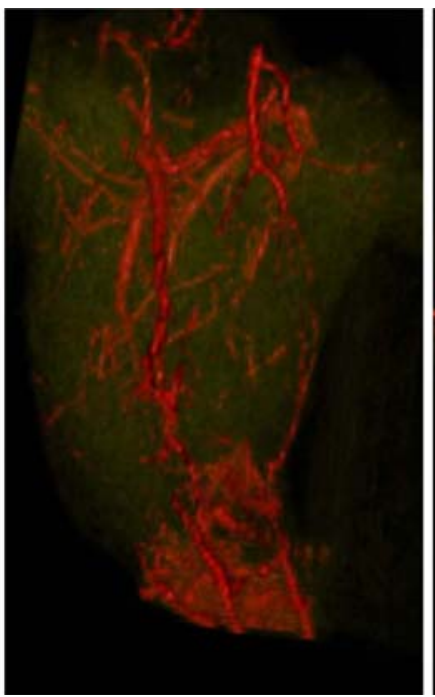

VEGF

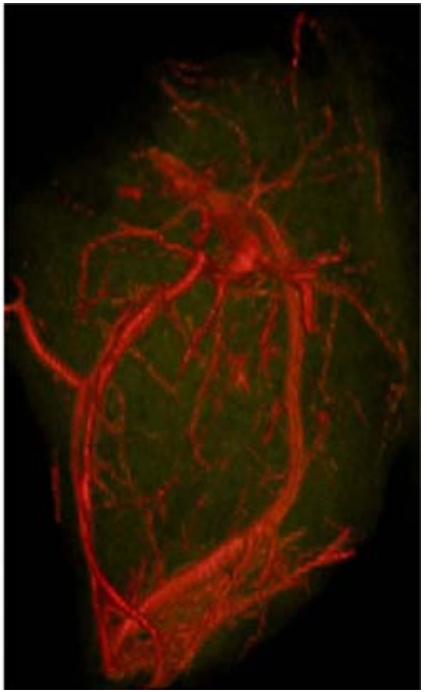

VEGF/PDGF

Fig. 4. Representative images of micro-CT after 5 weeks intramuscular injections of alginate (Blank), alginate containing $\mathrm{VEGF}_{165}$ (VEGF), or alginate containing $\mathrm{VEGF}_{165}$ combined with PLG microspheres containing PDGF-BB (VEGF/PDGF). 

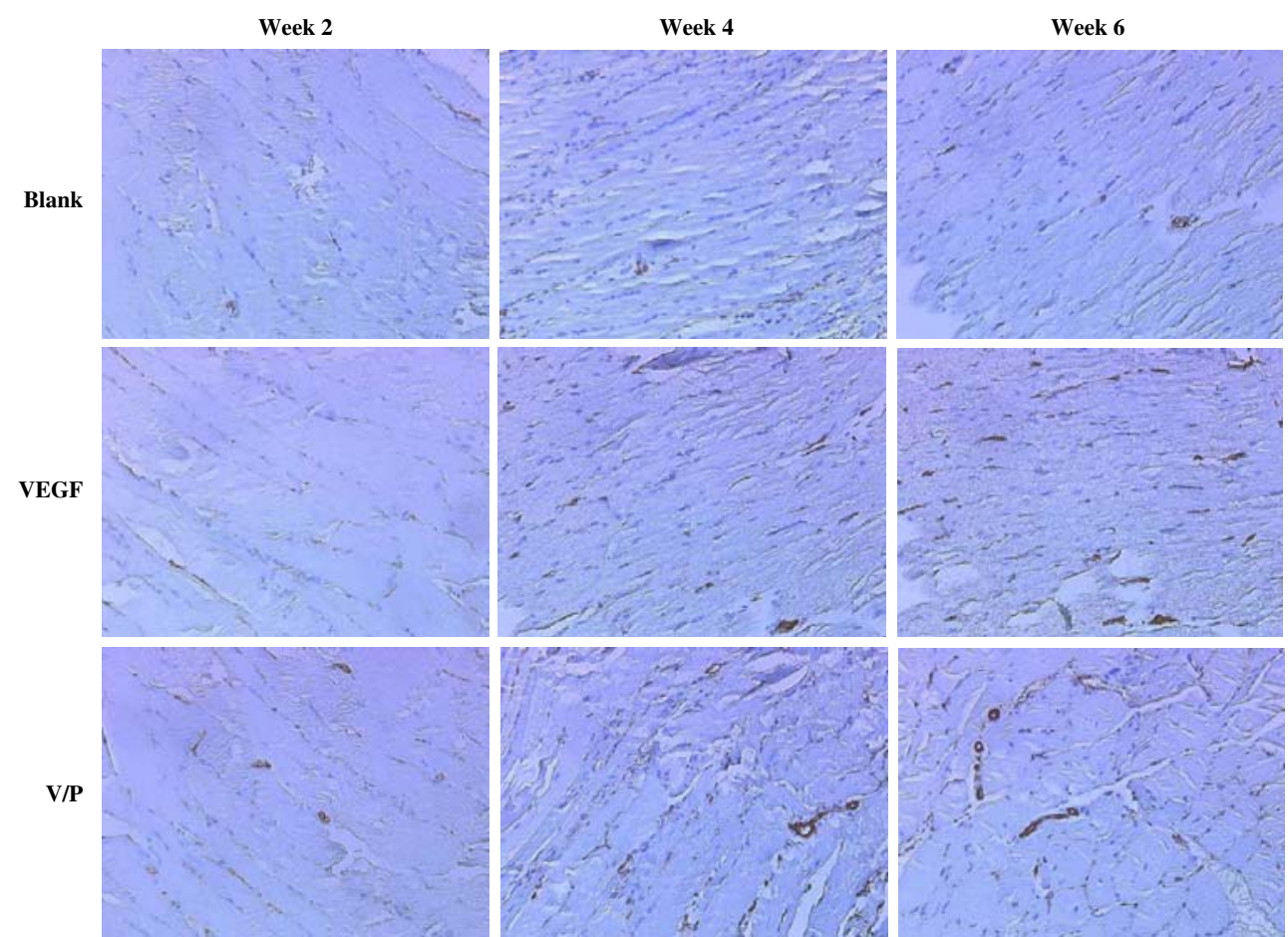

Fig. 5. Representative images of $\mathrm{CD} 31$ staining from thigh muscle of $a p o E^{-/-}$mice treated by intramuscular injections of alginate (Blank), alginate containing $\mathrm{VEGF}_{165}$ (VEGF), or alginate containing $\mathrm{VEGF}_{165}$ combined with PLG microspheres containing PDGF-BB (VEGF/PDGF) at 2, 4, and 6 weeks, $\times 200$.

required to compensate for occlusion of a single medium-size artery (13). Although whether VEGF is able to induce the growth of larger vessels (arterioles and arteries) is still unclear, VEGF seems to mediate the recruitment of circulat- ing monocytes that then stimulate a local arteriogenic process and play an important role in the maintenance of newly formed vasculature. There is evidence that newly formed vasculature in genetically altered mice completely disap-

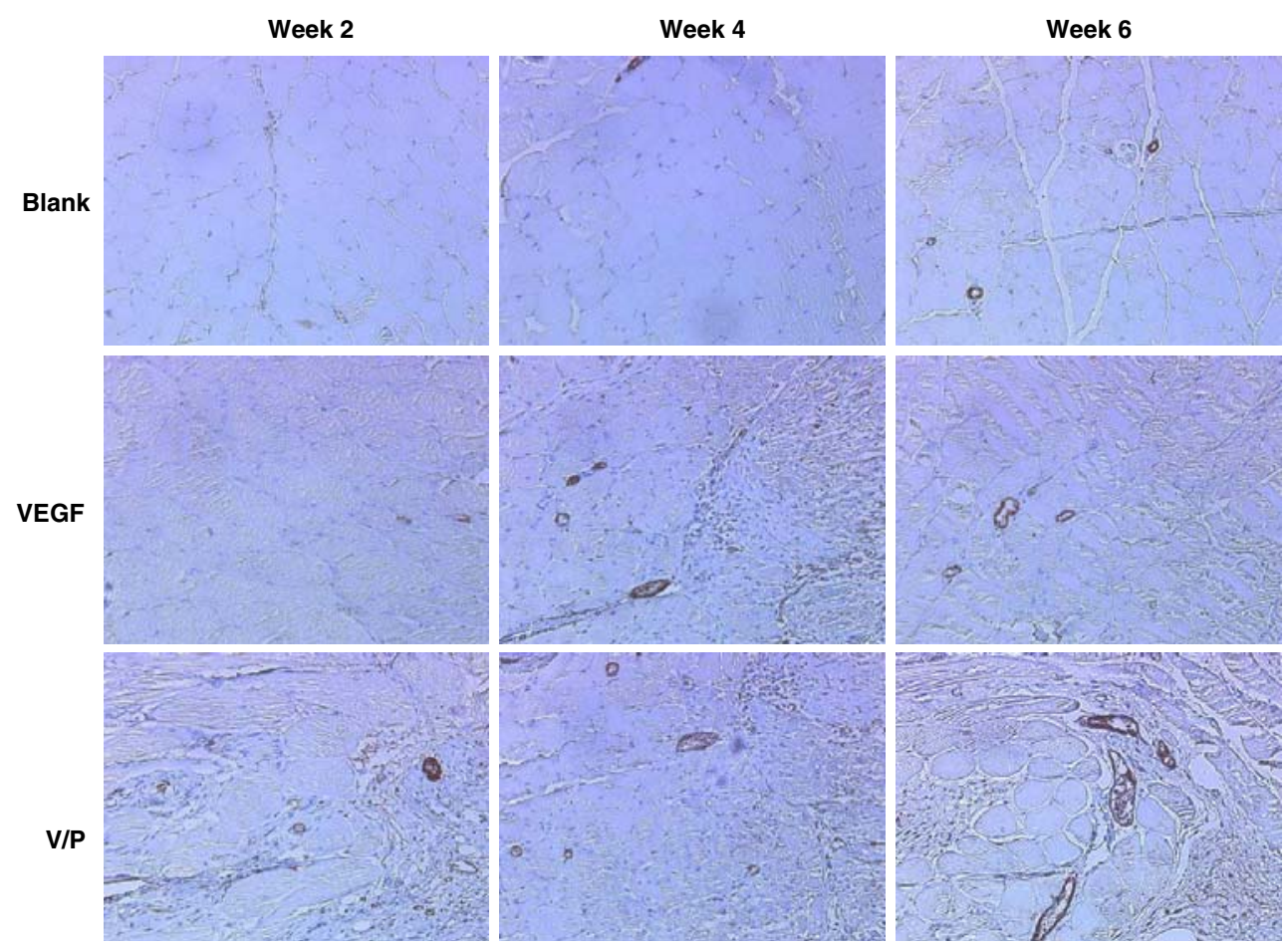

Fig. 6. Representative images of $\alpha$-SMA staining from thigh muscle of $a p o E^{-/}$mice treated by intramuscular injections of alginate (Blank), alginate containing $\mathrm{VEGF}_{165}$ (VEGF), or alginate containing $\mathrm{VEGF}_{165}$ combined with PLG microspheres containing PDGF-BB (VEGF/PDGF) at 2, 4, and 6 weeks, $\times 200$. 


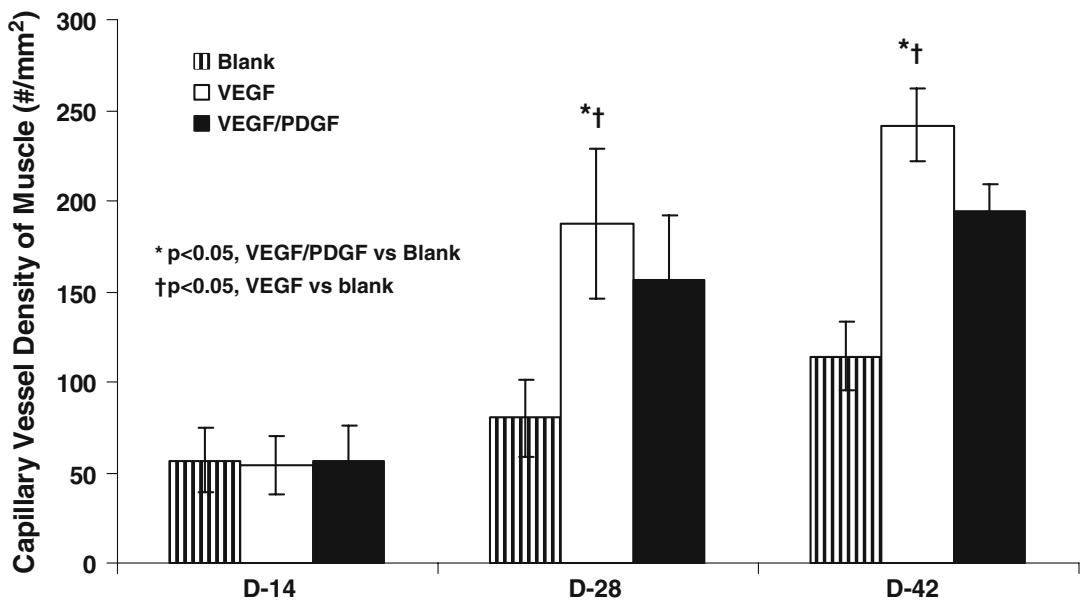

Fig. 7. Capillary vessel density from thigh muscle at 2, 4, and 6 weeks.

peared after a limited VEGF exposure, but that a longer duration of VEGF stimulation (10-14 days) resulted in the production of mature vessels even after withdrawal of the growth factor (14). In addition to VEGF, PDGF has been known to promote capillary growth leading to the development of larger vessels (15). Our data showed that a specific combination of these two angiogenic growth factors established functional and stable vascular networks and provided further information for the clinical trials for the treatment of vascular ischemic diseases.
The identification of growth factors involved in angiogenesis has led to their application in treating coronary artery disease and other diseases involving tissue ischemia. Bolus injections of solutions of growth factors have been investigated as treatments for myocardial ischemia, with promising results in animal models and small-scale clinical trials (16). However, these delivery methods did not appear to have as significant an effect in large clinical trials (17). This is likely related to the very short half-lives (minutes to hours) of these factors in vivo. One of the most important lessons from the

2 weeks

4 weeks

6 weeks
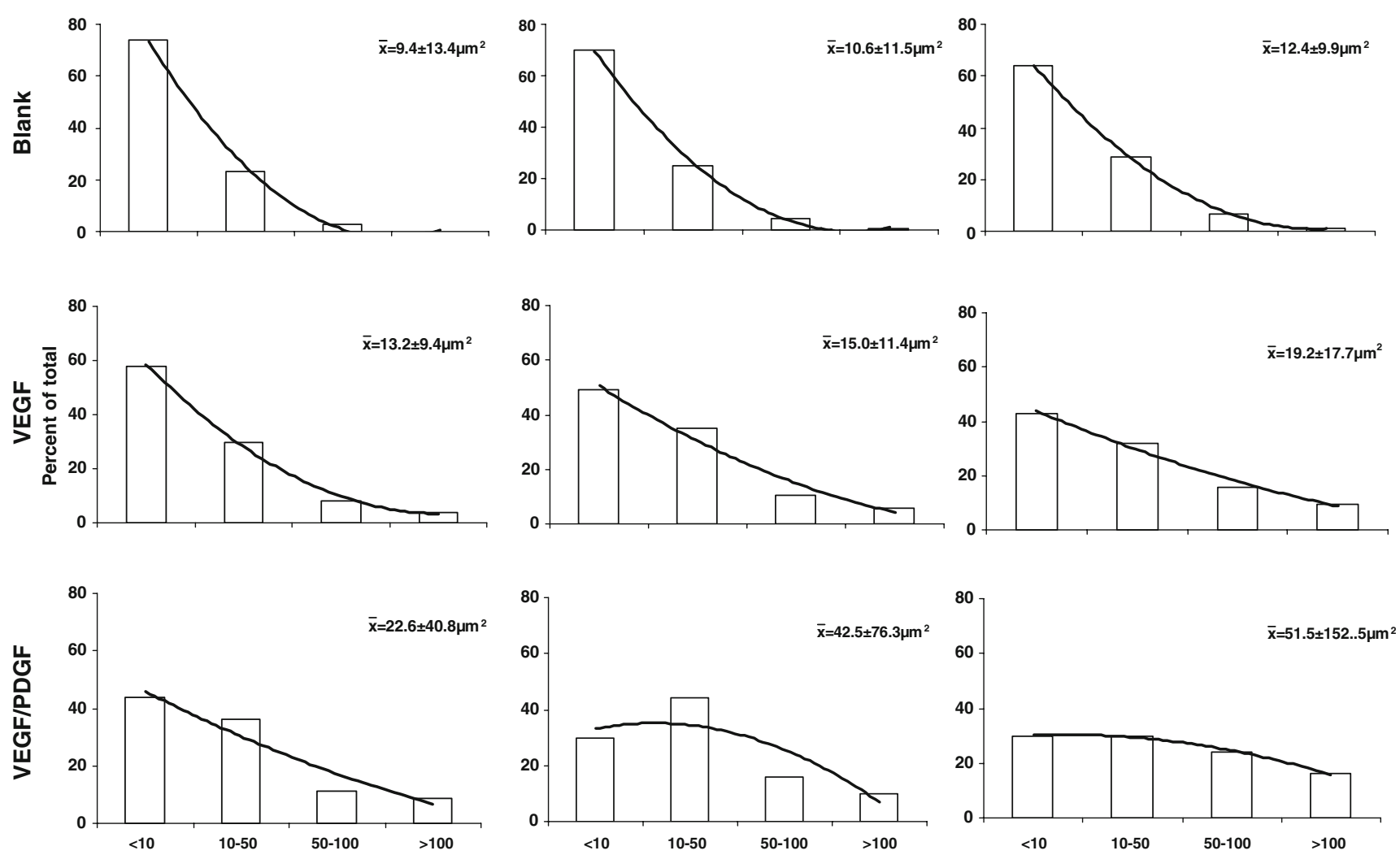

Fig. 8. Dual delivery of VEGF plus PDGF induced larger vessel formation. Cross-sectional areas of vessels were measured from $\alpha$-SMAstained thigh muscle of the mice treated by intramuscular injections of alginate (Blank), alginate containing VEGF $\mathrm{V}_{165}(\mathrm{VEGF})$, or alginate containing VEGF 165 combined with PLG microspheres containing PDGF-BB (VEGF/PDGF) at 2, 4, and 6 weeks. For each condition, at least 100 blood vessels from 6-10 tissue sections were counted. 
current generation of clinical trials, and ongoing basic research, has been the recognition of the need for prolonged tissue exposure to the growth factor for the development of robust and sustained neovascularization (18). Although a number of animal studies demonstrated the ability of a single bolus of growth factor to induce vigorous new vessel formation (19), such an approach has not been highly successful in clinical practice. One possible reason why bolus delivery of growth factor is not succeful in cinical trials is that the prolonged presence of a growth factor might be required to secure the survival of newly formed vasculature (14). Whether for tissue engineering or therapeutic applications, new vessels formed through the growth factor delivery strategies are typically small and lack pericyte incorporation. This finding suggests that they may not be functional longterm and may be subject to regression. A better, synchronized control of angiogenic cascade may be required for the formation of functional, stable vascular beds, since VEGF delivery followed by PDGF resulted in larger, mature vessel structures $(4,5)$. The theory is also confirmed by the current study, showing that the initial delivery of VEGF, following by a sustained release of PDGF, led to not only the formation but also the maturation of blood vessels. It is likely that the optimal time of exposure will differ according to the particular growth factor, formulation and delivery strategy, and so might need to be established for each combination.

Several important factors have to be considered in the design of a delivery system. First, it is essential to identify the key growth factor or factors to deliver for a particular tissue application, such as VEGF, FGF, and PDGF. The application of growth factors as therapeutic molecules has focused on those that are best characterized and available in large quantities as recombinant proteins. Second, the mode of factor delivery must target the desired cell population or tissue and minimize signal propagation to non-target cells and tissues. The application of growth factors involving intravenous injection is not localized to the target and is also ineffective because of the growth factors' short half-lives. Third, the controlled delivery of factors requires a relatively long-term maintenance of biologic activity within the system. Therefore, the method of fabrication that does not require harsh solvents or high temperatures is often desirable if the protein itself is used as the regenerative agent. Finally, the release profile of the growth factor from the system should be controlled temporally and spatially to be appropriate for a specific tissue injury or disease. Although numerous methods can be used to deliver sufficient amounts of growth factors to induce new vessel formation in animal models, the options in the clinic are very limited. The systemic administration to patients by intravenous infusion of angiogenic agents is complicated by dose-limiting hypotension and concerns regarding inappropriate angiogenesis resulting from systemic exposure. Therapeutic angiogenesis could increase a patient's future risk of cancer by creating an additional blood supply to small tumors in the body, thereby increasing their growth (18). It is possible that VEGF expression resulting from gene transfer could promote the development of a tumor that is too small to be recognized for months or even years (20). The polymeric delivery system with hydrogel and microspheres we used in the current study has several advantages. First, multiple growth factors or transplant cells that secrete the desired factor can be delivered. Second, the growth factors can be released in a controlled, sequenced, and time-dependent manner by controlled growth factor diffusion, polymer degradation, and the composition of the polymer. The combination of the different molecular weight polymers, both oxidized, allows us to create gels that degrade in vitro and in vivo in an appropriate time for drug delivery, while maintaining the ability of the system to form space filling gels that can provide a sustained release of a protein growth factor. Third, the delivery routes are very versatile, from topical to intra-operational, through local injection or through interventional catheter. Fourth, the delivery can be specifically targeted to prevent systemic side effects. Fifth, they are biocompatible. Such polymeric carriers have already obtained the Food and Drug Administration approval for the treatment of diabetic foot ulcers by PDGF delivery (21) and have been proved safe for patients. Various types of polymeric materials have been used for controlled release of bFGF and VEGF, including alginate hydrogels, PLG microspheres, and porous PLG scaffolds (22,23). Biodegradable synthetic polymers have found numerous applications of medical devices (e.g., sutures) due to the degradation into natural products of lactic and glycolic acid that enter into metabolic pathways. The physical properties of these polymers can also be readily altered by varying the ratio of lactide:glycolide, molecular weight, and crystallinity to meet various purposes in the clinic, and have the potential of flexibility to combine with other mediators to program angiogenic process and outcome (24-26).
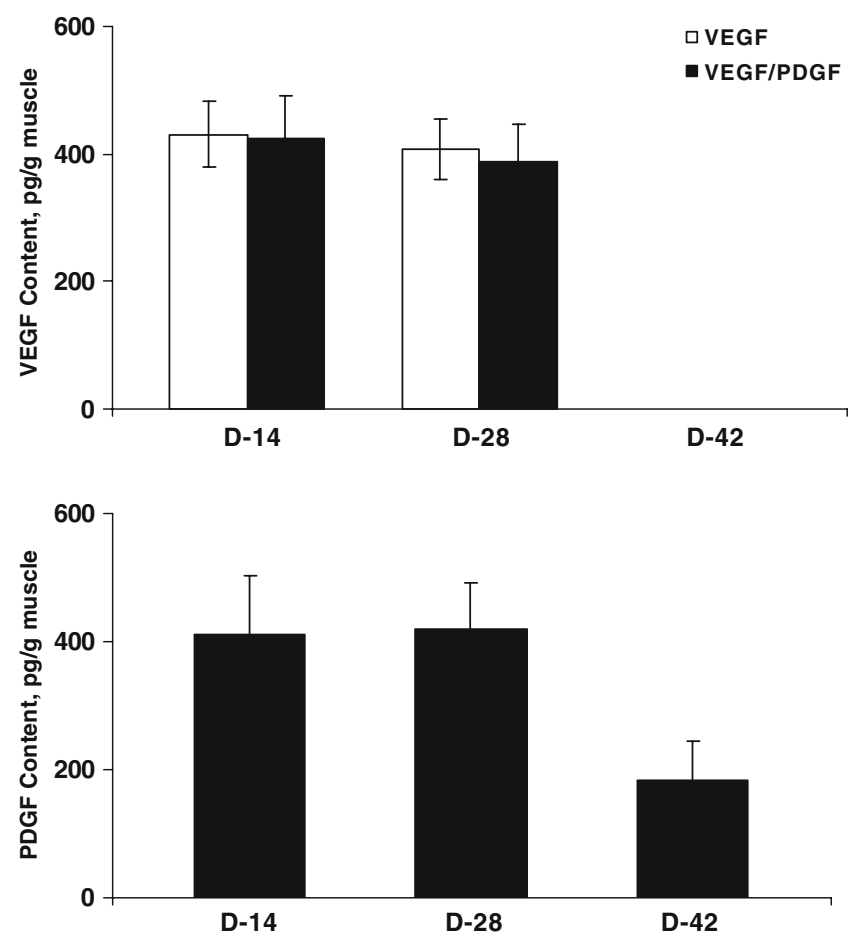

Fig. 9. VEGF concentration from thigh muscle of $\mathrm{apoE}^{-/-}$mice treated by alginate containing $\mathrm{VEGF}_{165}$ (VEGF), or alginate containing $\mathrm{VEGF}_{165}$ combined with PLG microspheres containing PDGF-BB (VEGF/PDGF); or PDGF concentration from thigh muscle treated by alginate containing $\mathrm{VEGF}_{165}$ combined with PLG microspheres containing PDGF-BB (VEGF/PDGF). 
Growth factor delivery is obviously a therapeutically important approach in proangiogenic experimental research and clinical application, such as cardiovascular regeneration. The limitation of single growth factor delivery has lead the way to the delivery strategy of multiple growth factors working in an orchestrated sequence to promote the regeneration of larger, stable vasculature. The current polymeric drug delivery system needs further investigation, including the appropriate dose, formulation, and administration route in both small and large animal models in order to determine how to achieve optimal myocardial and peripheral vascular angiogenesis, and hopefully opens a novel gateway for cardiovascular angiogenic therapy.

\section{ACKNOWLEDGEMENT}

This study was supported in part by NIH grant to Dr. Mooney (R01HL069957) and Dr. Rajagopalan (RO1ES015146). Dr. Sun was supported by NIH/NIEHS grant KO1ES016588.

Open Access This article is distributed under the terms of the Creative Commons Attribution Noncommercial License which permits any noncommercial use, distribution, and reproduction in any medium, provided the original author(s) and source are credited.

\section{REFERENCES}

1. Yancopoulos GD, Davis S, Gale NW, Rudge JS, Wiegand SJ, Holash J. Vascular-specific growth factors and blood vessel formation. Nature. 2000;407:242-8.

2. Hedman M, Hartikainen J, Syvanne M, Stjernvall J, Hedman A, Kivela A, et al. Safety and feasibility of catheter-based local intracoronary vascular endothelial growth factor gene transfer in the prevention of postangioplasty and in-stent restenosis and in the treatment of chronic myocardial ischemia: phase II results of the Kuopio Angiogenesis Trial (KAT). Circulation. 2003;107: 2677-83.

3. Henry TD, Annex BH, McKendall GR, Azrin MA, Lopez JJ, Giordano FJ, et al. The VIVA trial: vascular endothelial growth factor in ischemia for vascular angiogenesis. Circulation. 2003; 107:1359-65.

4. Richardson TP, Peters MC, Ennett AB, Mooney DJ. Polymeric system for dual growth factor delivery. Nat Biotechnol. 2001; 19:1029-34.

5. Sun Q, Chen RR, Shen Y, Mooney DJ, Rajagopalan S, Grossman PM. Sustained vascular endothelial growth factor delivery enhances angiogenesis and perfusion in ischemic hind limb. Pharm Res. 2005;22:1110-6.

6. Hao X, Silva EA, Mansson-Broberg A, Grinnemo KH, Siddiqui AJ, Dellgren G, et al. Angiogenic effects of sequential release of
VEGF-A165 and PDGF-BB with alginate hydrogels after myocardial infarction. Cardiovasc Res. 2007;75:178-85.

7. Mooney DJ, Kaufmann PM, Sano K, Schwendeman SP, Majahod $\mathrm{K}$, Schloo B, et al. Localized delivery of epidermal growth factor improves the survival of transplanted hepatocytes. Biotechnol Bioeng. 1996;50:422-9.

8. Couffinhal T, Silver M, Kearney M, Sullivan A, Witzenbichler B, Magner $\mathrm{M}$, et al. Impaired collateral vessel development associated with reduced expression of vascular endothelial growth factor in ApoE-/- mice. Circulation. 1999;99:3188-98.

9. Jorgensen SM, Demirkaya O, Ritman EL. Three-dimensional imaging of vasculature and parenchyma in intact rodent organs with X-ray micro-CT. Am J Physiol. 1998;275:H1103-14.

10. Sun Q, Yue P, Deiuliis JA, Lumeng CN, Kampfrath T, Mikolaj $\mathrm{MB}$, et al. Ambient air pollution exaggerates adipose inflammation and insulin resistance in a mouse model of diet-induced obesity. Circulation. 2009;119:538-46.

11. Simons M, Ware JA. Therapeutic angiogenesis in cardiovascular disease. Nat Rev Drug Discov. 2003;2:863-71.

12. Rajagopalan S, Grossman PM. Management of chronic critical limb ischemia. Cardiol Clin. 2002;20:535-45.

13. Helisch A, Schaper W. Arteriogenesis: the development and growth of collateral arteries. Microcirculation. 2003;10:83-97.

14. Dor Y, Djonov V, Abramovitch R, Itin A, Fishman GI, Carmeliet P, et al. Conditional switching of VEGF provides new insights into adult neovascularization and pro-angiogenic therapy. EMBO J. 2002;21:1939-47.

15. Cao R, Brakenhielm E, Pawliuk R, Wariaro D, Post MJ, Wahlberg E, et al. Angiogenic synergism, vascular stability and improvement of hind-limb ischemia by a combination of PDGFBB and FGF-2. Nat Med. 2003;9:604-13.

16. Freedman SB, Isner JM. Therapeutic angiogenesis for coronary artery disease. Ann Intern Med. 2002;136:54-71.

17. Laham R. Angiogenesis (clinical trials). Can J Cardiol. 2001;17 (Suppl A):29A-32A.

18. Jain RK, Carmeliet PF. Vessels of death or life. Sci Am. 2001; 285:38-45.

19. Post MJ, Laham R, Sellke FW, Simons M. Therapeutic angiogenesis in cardiology using protein formulations. Cardiovasc Res. 2001;49:522-31.

20. Grossman JD, Grossman W. Angiogenesis. Rev Cardiovasc Med. 2002;3:138-44.

21. Nagai MK, Embil JM. Becaplermin: recombinant platelet derived growth factor, a new treatment for healing diabetic foot ulcers. Expert Opin Biol Ther. 2002;2:211-8.

22. Ennett AB, Mooney DJ. Tissue engineering strategies for in vivo neovascularisation. Expert Opin Biol Ther. 2002;2:805-18.

23. Emerich DF, Mooney DJ, Storrie H, Babu RS, Kordower JH. Injectable hydrogels providing sustained delivery of vascular endothelial growth factor are neuroprotective in a rat model of Huntington's disease. Neurotox Res. 2009. doi:10.1007/s12640009-9079-0.

24. Discher DE, Mooney DJ, Zandstra PW. Growth factors, matrices, and forces combine and control stem cells. Science (New York, NY). 2009;324:1673-7.

25. Chan G, Mooney DJ. New materials for tissue engineering: towards greater control over the biological response. Trends Biotechnol. 2008;26:382-92.

26. Mooney DJ, Vandenburgh H. Cell delivery mechanisms for tissue repair. Cell Stem Cell. 2008;2:205-13. 